\title{
The Performance Evaluation of Transformation and Upgrading of Tourism Industry
}

\author{
Caixia Zhang ${ }^{1}$, Min Wei ${ }^{2}$ \\ ${ }^{1}$ Xiamen University Tan Kah Kee College, Zhangzhou 363105, China; \\ ${ }^{2}$ School of Management, Xiamen University, Xiamen 361005, China.
}

Keywords: Performance evaluation; Transformation and upgrading; Development; Tourism Industry.

\begin{abstract}
Tourism industry shows the features of the sustainable development. In order to drive the development of other industries, this paper aims to evaluate the performance of transformation and upgrading of tourism industry, including the rule of development, the past and present status as well as variation trends. The performance evaluation of transformation and upgrading of tourism industry reveals the future development changes according to law of development and trend of tourist market correlative variables to predict precisely. The practice based on historical data, the application of relevant theories, the dynamic mathematical model can be established to describe the transformation and upgrading of tourism industry, with the application of the related software to solve this mathematical model. Finally, Our results provide a new performance evaluation of transformation and upgrading of tourism industry.
\end{abstract}

\section{Introduction}

The researches and predictions of the performance evaluation of transformation and upgrading of tourism industry have been made some progress to solve the problems of the accuracy of numerical prediction to some extent. As we all know, the performance evaluation of transformation and upgrading of tourism industry includes three elements, the accuracy of the forecast values, the feasibility of forecasting and the operation of the predicting results. The prediction of the experts and scholars, however, pay too much attention to the accuracy of the forecast values neglecting the feasibility of forecasting and the operation of the predicting results. More importantly, it is very difficult to make stable value in terms of above predictive test method for testing the prediction, which is difficult to guarantee the feasibility of forecasting and the operation of the predicting results. Therefore, the theory and methods should be further amended above, as predicted for the unbalanced development of tourism industry. How to obtain stable, accurate predictive test value is very important [1]. This paper absorbs tourists in the tourism experience in forecasting drawn by experts in the field, applying Box-Jenkins time series modeling method, selecting a model with intercept term, as well as using to correct the prediction error. The serial correlation based on the application of simple linear regression model perspective, so as to provide scientific forecasts the tourism market testing methods. This method ensures not only the tourism market forecast accuracy and stability, but also the feasibility of forecasting and the operation of the predicting results.

\section{Literature Review}

Scholars carry out the exploration and research of tourism economy and industry forecasts based on various perspectives. The most frequently used models for forecasting with monthly data are time-series models, which use historical data to explain a variable and predict the future trend of the series. The most commonly used methods of forecasting the development of regional tourism industry are (seasonal) autoregressive (integrated) moving-average models ((S)AR(I)MA) [2]. Before 1990s, a combination of research method of the qualitative and quantitative is gradually accepted by most of researchers [3]. After1990s, with the maturation of artificial intelligence theory and its wide application in various industries, these methods will be gradually introduced to the prediction and evaluation of tourism market research. The focus of the tourism industry forecast is related tourism demand forecast. Unfortunately, 
most scholars focus more on the accuracy of numerical prediction and forecasting method of choice, instead of the feasibility and operability of prediction of the forecasting results.

Burger, Dohnal, Kathrada and Law make a brief description of the tourism attractions and context of Durban and South Africa to provide a qualitative feel of the system prior to the modeling process, by which they forecast the demand of tourism products/services in Durban and South Africa with some time series forecasting model, and then the appropriate conclusions can be drawn [4]. Planning for the future and forecasting what is likely to happen next stage of development, is crucial to the success of the whole tourism industry [5].

The tourism industry includes three elements, the accuracy of the forecast values, the feasibility of forecasting and the operation of the predicting results. The prediction of the experts and scholars, however, too much emphasis on the accuracy of the forecast values neglecting the feasibility of forecasting and the operation of the predicting results. More importantly, it is very difficult to make stable value in terms of above predictive test method for testing the prediction, which is difficult to guarantee the feasibility of forecasting and the operation of the predicting results. Therefore, the theory and methods should be further amended above, as predicted for the tourist market, how to obtain stable, accurate predictive test value is very important. This paper absorbed tourists in the performance evaluation of transformation and upgrading of tourism industry drawn by experts in the field, applied Box-Jenkins time series modeling method, selected a model with intercept term, used to correct the prediction error The serial correlation based on the application of simple linear regression model perspective, so as to provide scientific forecasts the tourism market testing methods. This method ensures not only the accuracy and stability of forecast for transformation and upgrading of tourism industry tourism industry, but also the feasibility of forecasting and the operation of the predicting results.

\section{Methodology}

With the arrival of the era of mass tourism, the development situation and tasks of tourism are undergoing profound changes. The problems such as insufficient effective supply of tourism, lagging public services, irregular market order, and inadaptable system and mechanism are becoming increasingly prominent. It is urgent to innovate the concept of development, accelerate the transformation and upgrading of tourism, improve quality and efficiency. The Guiding Opinion states that the development of tourism in the whole region, taking a certain region as a complete tourist destination, taking tourism as a dominant industry, unified planning and distribution, optimizing public services, promoting industrial integration, strengthening comprehensive management, and implementing systematic marketing can continuously promote the modernization, intensiveness, quality and internationalization of tourism. To maximize the satisfaction of tourism consumption and effectively promote economic and social development.

This paper formulates and improves Yunnan standards in the fields of tourism product format, tourism factor facilities, tourism public service, industrial operation management, market supervision and management, and serves as the operational guide for tourism service providers and the basis for evaluation and management of government departments and trade associations. The current time $t$ for the sequence $X_{t}$ to predict the observed values $X_{t+l}$ of the relevant variables in the transformation and upgrading of tourism industry $t$ to moment $t+l$, the transformation and upgrading of tourism industry is called thought the origin $t$ to the pre- (or step ) $l$ for the prediction, with predictive value $\hat{X}_{t}(l)$ denoted. The main idea of the transformation and upgrading of tourism industry is to regard sequence at the time $t+l$ of the conditional expectation $X_{t+l}$ as the time $t+l$ of the transformation and upgrading of tourism industry value $X_{t+l}$.In the model $\operatorname{ARMA}(p, q)$, the conditional transformation and upgrading of tourism industry of time series $X_{t}$ and random disturbance $\alpha_{t}$ have the following characteristics:(1) Conditional expectation of the observational value for the current time and past time is for itself;(2) Conditional expectation of the transformation and upgrading of tourism industry value for the current 
time and past time is for itself;(3)Transformation and upgrading of tourism industry of the random perturbations value for the future continuous time is zero;(4)Transformation and upgrading of tourism industry for the future continuous time.

According to the transformation and upgrading of tourism industry $\operatorname{ARMA}(p, q)$, the time $t+l$ of the sequence value $X_{t+l}$ of the model can be obtained as below Equation (1), with the time of transformation and upgrading of tourism industry is replaced.

$$
X_{t+l}=\varphi_{1} X_{t+l-1}+\varphi_{2} X_{t+l-2}+\cdots+\varphi_{p} X_{t+l-p}+\alpha_{t+l}-\theta_{1} \alpha_{t+l-1}-\theta_{2} \alpha_{t+l-2}-\cdots-\theta_{q} \alpha_{t+l-q}
$$

Similarly, based on Equation (1), with the utilization of the transformation and upgrading of tourism industry, and the value $X_{t+l}$ for the time $t+l$ can be calculated as shown in Equation (2).

$$
X_{t+l}^{\prime}=\varphi_{1} X_{t+l-1}^{\prime}+\varphi_{2} X_{t+l-2}^{\prime}+\cdots+\varphi_{p} X_{t+l-p}^{\prime}+\alpha_{t+l}^{\prime}-\theta_{1} \alpha_{t+l-1}^{\prime}-\theta_{2} \alpha_{t+l-2}^{\prime}-\cdots-\theta_{q} \alpha_{t+l-q}^{\prime}
$$

\section{Results and analyses}

With the guidance of the government and the main body of the enterprise as the main means, we should encourage and support the deep integration project of the whole region's tourism convergence application, large data and the real economy of the tourism through PPP mode, BT mode, enterprises' self-investment and self-construction and self-operation, service purchase, resource replacement, market operation, market investment and financing, etc. Land-based construction, cultivate the benign "hematopoietic" function of enterprises, form a large tourism data business model, improve the sustainability of large tourism data.

Focusing on building a "one-stop" service platform for intelligent tourism, we should deepen the capacity building of large data in the field of tourism, build a comprehensive analysis and management system for large data of tourism in the whole region, accelerate the formation of guiding norms for intelligent tourist attractions, and gradually expand the coverage. Through the implementation of pilot demonstration projects, cultivate a number of tourism and large data deep integration of typical enterprises, forming a number of advanced technology, products, models, to promote the application of large data in the tourism industry. Encourage tourism departments at all levels, tourist attractions, travel agencies, hotels, farmhouse entertainment, etc. to fully consider the needs of large data acquisition in the information construction, build a hierarchical and classified tourism large data resources system, implement the opening of tourism public data resources, guide the development of tourism large data resources and market-oriented operation, and effectively To integrate data sharing and exchange among travel departments, industries and application systems.

In fact, the core of the "transformation" in the transformation and upgrading of industrial structure is to change the "type" of economic growth, that is, to turn high input, high consumption, high pollution, low output, low quality and low efficiency into low input, low consumption, low pollution, high output, high quality and high efficiency, and to turn extensive into intensive rather than intensive. Simple transfer industry. There is no necessary link between industry transformation and transformation. It is not necessarily possible to transform the industry if the industry is changed, and it is not necessary to change the industry if the industry is to be transformed. The "upgrading" in the transformation and upgrading of industrial structure includes not only the upgrading between industries, such as the gradual evolution from the dominant proportion of the primary industry to the dominant proportion of the secondary and tertiary industries in the whole industrial structure, but also the upgrading within the industry, i.e. the gradual deepening development of the processing and re-engineering system within a certain industry. Technology intensification and continuous improvement of production efficiency. Only by correctly understanding these connotations of transformation and upgrading of industrial structure can we avoid deviations in practice. Both predictive accuracy test of upgrading of tourism industry can be expressed mathematically that whether the average number of series $\left(d_{t}\right)$ formed by different model prediction error function is 0 or not. The inspection process is shown in Fig. 1: 


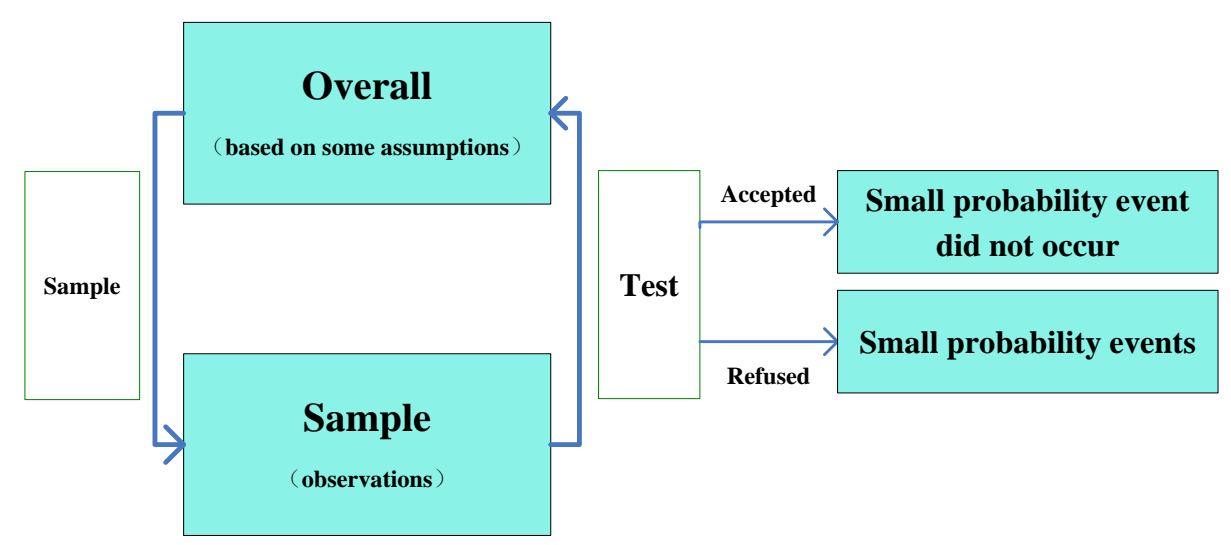

Fig. 1 Tourism market forecasting performance testing program

\section{Conclusion}

This paper attempts to improve the performance evaluation of transformation and upgrading of tourism industry, because the forecasts of current tourism industry, ignoring the importance of the feasibility and operability for the predicted results of the unbalanced development of tourism industry, as well as trying to enrich and to develop forecast theory of the tourism industry. The tourism industry forecast is a new topic of the global scope, but also it is the key of comprehensively implement the scientific concept of development, the harmonious development of man and nature co-ordinate. Therefore, simple linear regression model proposed in this new perspective is applied to the analysis of the tourism market forecast study. Taking into account the accuracy of the forecast value, forecast the results of the feasibility and operability of prediction, it can be considered to be more effectively guide the development of the tourism market practice. The research results are not only to provide a new performance evaluation of transformation and upgrading of tourism industry, but also to try to provide a theoretical basis and method selection for the country and the global tourism market forecasts by drawing general rules.

\section{References}

[1]. Song, H., Smeral, E, Li, G., and Chen, J.L., “Tourism forecasting using econometric models,” Trends in European tourism planning and organisation, Channel View, 2013, pp.289-309.

[2]. Kim, N., \& Schwartz, Z, "The accuracy of tourism forecasting and data characteristics: a meta-analytical approach,” Journal of Hospitality Marketing \& Management, vol.22, 2013, pp.349-374.

[3]. Doan, T.A., Litterman, R.B., and Sims, C.A., "Forecasting and conditional projections using realistic prior distributions,” Econometric Reviews,1984, vol.1, pp.1-10.

[4]. Burger, C., Dohnal, M., Kathrada, M., and Law, R, “A practitioners guide to time-series methods for tourism demand forecasting — a case study of Durban, South Africa,” Tourism Management, 2001, vol.22(4), pp.403-409.

[5]. Gunter, U., and Önder I, "Forecasting international city tourism demand for Paris: Accuracy of uniand multivariate models employing monthly data,” Tourism Management, 2015, vol.46, pp.123-135. 